\title{
Normal Form for Systems with Linear Part $N_{3(n)}$
}

\author{
Grace Gachigua $^{1 *}$, David Malonza ${ }^{2}$, Johana Sigey ${ }^{3}$ \\ ${ }^{1}$ Department of Mathematics, College of Technology, Kimathi University, Nyeri, Kenya \\ ${ }^{2}$ Department of Mathematics, Kenyatta University, Nairobi, Kenya \\ ${ }^{3}$ Pure and Applied Mathematics (PAM) Department, Jomo Kenyatta University of Agriculture and Technology, Nairobi, Kenya \\ Email: *wambuigachigua@gmail.com,dmalo2004@gmail.com,jksigey2002@yahoo.com
}

Received August 10, 2012; revised September 17, 2012; accepted September 24, 2012

\begin{abstract}
The concept of normal form is used to study the dynamics of non-linear systems. In this work we describe the normal form for vector fields on $\mathbb{R}^{3 n}$ with linear nilpotent part made up of coupled $n 3 \times 3$ Jordan blocks. We use an algorithm based on the notion of transvectants from classical invariant theory known as boosting to equivariants in determining the normal form when the Stanley decomposition for the ring of invariants is known.
\end{abstract}

Keywords: Transvectant; Equivariants; Box Product; Stanley Decomposition

\section{Introduction}

There are well-known procedures for putting a system of differential equations $\dot{x}=A x+v(x)$ (where $v$ is a formal power series starting with quadratic terms) into normal form with respect to its linear part A. Our concern in this paper is to describe the normal form of the systemm $\dot{x}=A x+v(x)$, that is the set of all $v$ such that $A x+v(x)$ is in normal form where $A$ is the linear part $N_{33, \cdots, 3}$ from the Stanley decomposition of the ring of invariants. Our main result is a procedure that solves the description problem where $N$ is a nilpotent matrix with coupled n Jordan blocks, provided that the description problem is already solved for each Jordan block of $N$ taken separately. Our method is based on adding one block at a time. This procedure will be illustrated with examples and then be generalized.

The idea of simplification near an equilibrium goes back at least to Poincare (1880), who was among the first to bring forth the theory in a more definite form. Poincare considered the problem of reducing a system of nonlinear differential equations to a system of linear ones. The formal solution of this problem entails finding nearidentity coordinate transformations, which eliminate the analytic expressions of the nonlinear terms.

Cushman et al. [1], using a method called covariant of special equivariant solved the problem of finding Stanley decomposition of $N_{22, \ldots, 2}$. Their method begins by creating a scalar problem that is larger than the vector problem and their procedures are derived from classical invariant theory thus it was necessary to repeat calculations of classical invariants theory at the levels of equi-

\footnotetext{
*Corresponding author.
}

variants. Malonza [2] solved the same problem by "Groebner" basis methods found in [3] rather than borrowing from classical theory.

Murdock and Sanders [4] developed an algorithm based on the notion of transventants to determine the form of normal form of a vector field with nilpotent linear part, when the normal form is known for each Jordan block of the linear part taken separately. The algorithm is based on the notion of transvectants from the classical invariant theory known as boosting to module of equivariants when the Stanley decomposition for the ring of invariants is known.

Namachchivaya et al. [5], studied a generalized Hopf bifurcation with non-semisimple 1:1 Resonance. The normal form for such a system contains only terms that belong to both the semisimple part of $\mathrm{A}$ and the normal form of the nilpotent, which is a coupled TakensBogdanov system with $A=\left[\begin{array}{cccc}i \omega & 1 & & \\ & i \omega & & \\ & & i \omega & 1 \\ & & & i \omega\end{array}\right]$.

This example illustrates the physical significance of the study of normal forms for systems with nilpotent linear part.

Our results are mainly based on the work found in [4] that is application of transvectant's method for computing normal form for the module of equivariants of nilpotent systems. In section two and three we put together background knowledge for understanding the content of this work. Section four forms the central part of this paper where we shall compute the module of equivariants. 


\section{Invariants and Stanley Decompositions}

Let $\mathcal{P}_{j}\left(\mathbb{R}^{n}, \mathbb{R}^{m}\right)$ denote the vector space of homogeneous polynomials of degree $j$ on $\mathbb{R}^{n}$ with coefficients in $\mathbb{R}^{m}$, where $\mathbb{R}$ denotes the set of real numbers. Let $\mathcal{P}\left(\mathbb{R}^{n}, \mathbb{R}^{m}\right)$ be the vector space of all such polynomials of any degree and let $\mathcal{P}_{*}\left(\mathbb{R}^{n}, \mathbb{R}^{m}\right)$ be the vector space of formal power series. If $m=1, \mathcal{P}_{*}\left(\mathbb{R}^{n}, \mathbb{R}^{m}\right)$ becomes the ring of formal power series on $\mathbb{R}^{n}$, where $\mathbb{R}$ denotes the set of real numbers. For such smooth vectors fields, it is sufficient to work polynomials. For any nilpotent matrix $N$, we define the Lie operator

$$
L_{N}: \mathcal{P}_{j}\left(\mathbb{R}^{n}, \mathbb{R}^{n}\right) \rightarrow \mathcal{P}_{j}\left(\mathbb{R}^{n}, \mathbb{R}^{n}\right)
$$

by

$$
\left(L_{N} v\right) x=v^{\prime}(x) N x-N v(x)
$$

and the differential operator

$$
\mathcal{D}_{N x}: \mathcal{P}_{j}\left(\mathbb{R}^{n}, \mathbb{R}\right) \rightarrow \mathcal{P}_{j}\left(\mathbb{R}^{n}, \mathbb{R}\right)
$$

by

$$
\left(\mathcal{D}_{N x} f\right)(x)=f^{\prime}(x) N x=(N x . \nabla) f(x) .
$$

Then $\mathcal{D}_{N}$ is a derivation of the ring $\mathcal{P}\left(\mathbb{R}^{n}, \mathbb{R}\right)$, meaning that

$$
\mathcal{D}(f g)=\left(\mathcal{D}_{N} f\right) g+f \mathcal{D}_{N} g .
$$

In addition,

$$
L_{N}(f v)=\left(\mathcal{D}_{N} f\right) v+f L_{N} v .
$$

A function $f$ is called an invariant of $A x$ if $\left.\frac{\partial}{\partial t} f\left(e^{A t} x\right)\right|_{t=0}=0$ or equivalently $f \in \operatorname{ker} \mathcal{D}_{A}$. Since

$$
\begin{gathered}
\mathcal{D}_{N}(f+g)=\mathcal{D}_{N} f+\mathcal{D}_{N} g \\
\mathcal{D}_{N} f g=f \mathcal{D}_{N} g+g \mathcal{D}_{N} f
\end{gathered}
$$

it follows that if $f$ and $g$ are invariants, so are $f+g$ amd $f g$; that is $\operatorname{ker} \mathcal{D}_{N}$ is both a vector space over $\mathbb{R}$ and also a subring of $\mathcal{P}\left(\mathbb{R}^{n}, \mathbb{R}\right)$, known as the ring of invariants. Similarly a vector field $v$ is called an equivariants of $A x$, if $\left.\frac{\partial}{\partial t}\left(e^{-A t} v\left(e^{A t} x\right)\right)\right|_{t=0}=0$ that is $v \in \operatorname{ker} L_{A}$.

There are two normal form styles in common use for nilpotent systems, the inner product normal form and the sl(2) normal form. The inner product normal form is defined by $\mathcal{P}\left(\mathbb{R}^{n}, \mathbb{R}\right)=i m L_{N} \oplus \operatorname{ker} L_{N^{*}}$ where $N^{*}$ is the conjugate transpose of $N$. To define the $s l(2)$ normal form, one first sets $X=N$ and constructs matrices $Y$ and $Z$ such that

$$
[X, Y]=Z, \quad[Z, X]=2 X, \quad[Z, Y]=-2 Y .
$$

An example of such an $\operatorname{sl}(2)$ triad $\{X, Y, Z\}$ is

$$
\begin{aligned}
& X=\left[\begin{array}{llll}
0 & 1 & 0 & 0 \\
0 & 0 & 0 & 0 \\
0 & 0 & 0 & 1 \\
0 & 0 & 0 & 0
\end{array}\right], \quad Y=\left[\begin{array}{llll}
0 & 0 & 0 & 0 \\
1 & 0 & 0 & 0 \\
0 & 0 & 0 & 0 \\
0 & 0 & 1 & 0
\end{array}\right], \\
& Z=\left[\begin{array}{cccc}
1 & 0 & 0 & 0 \\
0 & -1 & 0 & 0 \\
0 & 0 & 1 & 0 \\
0 & 0 & 0 & -1
\end{array}\right]
\end{aligned}
$$

Having obtained the triad $\{X, Y, Z\}$ we create two additional triads $\{\mathcal{X}, \mathcal{Y}, \mathcal{Z}\}$ and $\{\mathrm{X}, \mathrm{Y}, \mathrm{Z}\}$ as follows

$$
\begin{aligned}
& \mathcal{X}=\mathcal{D}_{Y}, \quad \mathcal{Y}=\mathcal{D}_{X}, \quad \mathcal{Z}=\mathcal{D}_{Z} \\
& X=L_{Y}, \quad Y=L_{X}, \quad Z=L_{Z}
\end{aligned}
$$

The first of these is a triad of differential operators and the second is a triad of Lie operators. Both the operators $\{\mathcal{X}, \mathcal{Y}, \mathcal{Z}\}$ and $\{\mathrm{X}, \mathrm{Y}, \mathrm{Z}\}$ inherit the triad properties (2.5). Observe that the operators $\{X, Y, Z\}$ map each $\mathcal{P}\left(\mathbb{R}^{n}, \mathbb{R}^{n}\right)$ into itself. It follows from the representation theory $\operatorname{sl}(2)$ that

$$
\mathcal{P}\left(\mathbb{R}^{n}, \mathbb{R}^{n}\right)=\operatorname{im} Y \oplus \operatorname{ker} \mathrm{X}=\operatorname{im} \mathrm{X} \oplus \operatorname{ker} \mathrm{M}
$$

Clearly the ker $\mathcal{X}$ ia $\mathrm{s}$ subring of $\mathcal{P}\left(\mathbb{R}^{n}, \mathbb{R}\right)$, the ring of invariants and it follows from (2.4) that ker $X$ is a module over this subring. This is the sl(2) normal form module.

\section{Boosting Rings of Invariants to Module of Equivariants}

In this section we describe the procedure for obtaining a Stanley decomposition of the module of equivariants (or normal form space ker $X$ ) when the Stanley decomposition of the ring of invariants is known.

The module of all formal power series vector fields on $\mathbb{R}^{n}$ can be viewed as the tensor product

$\mathbb{R}\left[\left[x_{1}, \cdots, x_{n}\right]\right] \otimes \mathbb{R}^{n}$, and in fact the tensor product can be identified with the ordinary product (of a field times a constant vector) since the ordinary product satisfies the same algebraic rules as a tensor product. Specifically, every formal power series vector field can be written as

$$
f_{1}(x) e_{1}+\ldots+f_{n}(x) e_{n}=\left[\begin{array}{c}
f_{1}(x) \\
\vdots \\
f_{n}(x)
\end{array}\right]
$$

where the $e_{i}$ are the standard basis vectors of $\mathbb{R}^{n}$. Next, the Lie derivative $X=L_{N^{*}}$ can be expressed as the tensor product of $\mathcal{X}$ and $-N^{*}$, that is

$\mathrm{X}=\mathcal{X} \otimes I+I \otimes\left(-N^{*}\right)$. Under the identification of $\otimes$ 
with ordinary product, this means

$X(f v)=(\mathcal{X} f) v+f\left(-N^{*}\right)$, where $f \in \mathbb{R}\left[\left[x_{1}, \cdots, x_{n}\right]\right]$ and $v \in \mathbb{R}^{n}$ in agreement with the following calculation, in which $v^{\prime}=0$ because $v$ is constant.

$$
\begin{aligned}
X(f v) & =L_{N^{*}}(f v)=\left(\mathcal{D}_{N^{*}} f\right) v+f\left(L_{N^{*}} v\right) \\
& =\left(\mathcal{D}_{N^{*}} f\right) v+f\left(v^{\prime} N^{*} x-N^{*} v\right) \\
& =\left(\mathcal{D}_{N^{*}}\right) v+f\left(-N^{*} v\right) .
\end{aligned}
$$

This kind of calculation also shows that $\operatorname{sl}(2)$ representation (on vector fields ) with triad $\{\mathrm{X}, \mathrm{Y}, \mathrm{Z}\}$ is the tensor product of the representation (on scalar fields) with triad $\{\mathcal{X}, \mathcal{Y}, \mathcal{Z}\}$ and the representation (on $\mathbb{R}^{*}$ with triad $\left\{-N^{*},-M^{*},-H\right\}$ that is

$$
\text { ker } \mathrm{X}=\operatorname{ker} \mathcal{X} \otimes \mathbb{R} e_{r} \text {. }
$$

It follows that a basis from the normal form space ker $X$ is given by well defined transvectants $(f, v)^{(i)}$ as $f$ ranges over a basis for ker $\mathcal{X} \subset \mathbb{R}\left[\left[x_{1}, \cdots, x_{n}\right]\right]$ and $v$ ranges over a basis for $\operatorname{ker} N^{*} \subset \mathbb{R}^{n}$. The first of these bases is given by the standard monomials of a Stanley decomposition for ker $\mathcal{X}$. The second is given by the standard basis vectors $e_{r} \in \mathbb{R}$ such that $r$ is the index of the bottom row of a Jordan block in $N$. It is useful to note that the weight of such an $e_{r}$ is one less than the size of the block. Then we define the transvectant $(f, v)^{i}$ as

$$
\begin{aligned}
\left(f, e_{r}\right)^{i} & =\sum_{j=0}^{i}(-1)^{j} W_{f, e_{r}}^{i, j}\left(\mathcal{Y}^{j} f\right)\left(\left(-M^{*}\right)^{j-1} e_{r}\right) \\
& =(f, g)^{i} \\
& =\sum_{j=0}^{i}(-1)^{j} W_{f, g}^{i, j}\left(\mathcal{Y}^{j} f\right)\left(\left(M^{*}\right)^{j-1} g\right) .
\end{aligned}
$$

From here, the computational procedures of box products are the same as those used in describing rings of invariants from [4], except that infinite iterations never arise.

\section{Normal Form for Systems with Linear Part $N_{3(n)}$}

Before generalizing we shall consider the normal form for nonlinear systems with linear part having two and three blocks, that is $N_{33}$ and $N_{333}$ as examples.

\subsection{System with Linear Part $N_{33}$}

The Stanley decomposition for the ring of invariants with linear part $N_{33}$ is given by:

$$
\begin{aligned}
& \operatorname{ker} \mathcal{X}_{33}= \mathbb{R}\left[\left[\alpha_{1}, \alpha_{2}, \beta_{1}, \beta_{2}, \xi_{1,2}\right]\right] \\
& \oplus \mathbb{R}\left[\left[\alpha_{1}, \alpha_{2}, \beta_{1}, \beta_{2}, \xi_{1,2}\right]\right] \gamma_{1,2}
\end{aligned}
$$

(see [6]). Since $\beta_{1}, \beta_{2}$ and $\xi_{1,2}$ has weight zero, it is convenient to remove them since we do not expand along terms of weight zero by setting $\mathcal{R}=\left[\left[\beta_{1}, \beta_{2}, \xi_{1,2}\right]\right]$ and write

$$
\begin{aligned}
\operatorname{ker} \mathcal{X}_{33} & =\mathcal{R}\left[\left[\alpha_{1}, \alpha_{2}\right]\right] \oplus \mathcal{R}\left[\left[\alpha_{1}, \alpha_{2}\right]\right] \gamma_{1,2} \\
& =\mathcal{R}\left[\left[\alpha_{2}\right]\right] \oplus \mathcal{R}\left[\left[\alpha_{1}, \alpha_{2}\right]\right] \alpha_{1} \oplus \mathcal{R}\left[\left[\alpha_{1}, \alpha_{2}\right]\right] \gamma_{1,2} \\
& =\mathcal{R} \oplus \mathcal{R}\left[\left[\alpha_{2}\right]\right] \alpha_{2} \oplus \mathcal{R}\left[\left[\alpha_{1}, \alpha_{2}\right]\right] \alpha_{1} \\
& \oplus \mathcal{R}\left[\left[\alpha_{1}, \alpha_{2}\right]\right] \gamma_{1,2}
\end{aligned}
$$

In this case the basis elements are $e_{3}$ and $e_{6}$. Therefore we need to compute the box product of the ring ker $\mathcal{X}_{33}$ with $\mathbb{R} e_{3} \oplus \mathbb{R} e_{6}$ which are both of weight 2 .

Therefore $\quad \operatorname{ker} \mathrm{X}_{33}=\left(\operatorname{ker} \mathcal{X}_{33}\right) \otimes\left(\mathbb{R} e_{3} \oplus \mathbb{R} e_{6}\right)$. Distributing the box product there are two cases to consider.

Case 1:

$$
\begin{aligned}
& \mathcal{R} \oplus \mathcal{R}\left[\left[\alpha_{2}\right]\right] \alpha_{2} \oplus \mathcal{R}\left[\left[\alpha_{1}, \alpha_{2}\right]\right] \alpha_{1} \\
& \oplus \mathcal{R}\left[\left[\alpha_{1}, \alpha_{2}\right]\right] \gamma_{1,2} \otimes \mathbb{R} e_{3}
\end{aligned}
$$

There are four products namely:

a) $\mathcal{R} \otimes \mathbb{R} e_{3}=\mathcal{R} e_{3}$

b)

$$
\begin{aligned}
& \mathcal{R}\left[\left[\alpha_{2}\right]\right] \alpha_{2} \otimes \mathbb{R} e_{3}=\mathcal{R}\left[\left[\alpha_{2}\right]\right] \alpha_{2} e_{3} \\
& \oplus \mathcal{R}\left[\left[\alpha_{2}\right]\right]\left(\alpha_{2}, e_{3}\right)^{(1)} \oplus \mathcal{R}\left[\left[\alpha_{2}\right]\right]\left(\alpha_{2}, e_{3}\right)^{(2)} \\
& \mathcal{R}\left[\left[\alpha_{1}, \alpha_{2}\right]\right] \alpha_{1} \otimes \mathbb{R} e_{3}=\mathcal{R}\left[\left[\alpha_{1}, \alpha_{2}\right]\right] \alpha_{1} e_{3} \\
& \oplus \mathcal{R}\left[\left[\alpha_{1}, \alpha_{2}\right]\right]\left(\alpha_{1}, e_{3}\right)^{(1)} \oplus \mathcal{R}\left[\left[\alpha_{1}, \alpha_{2}\right]\right]\left(\alpha_{1}, e_{3}\right)^{(2)} \\
& \mathcal{R}\left[\left[\alpha_{1}, \alpha_{2}\right]\right] \gamma_{1,2} \otimes \mathbb{R} e_{3}=\mathcal{R}\left[\left[\alpha_{1}, \alpha_{2}\right]\right] \gamma_{1,2} e_{3} \\
& \oplus \mathcal{R}\left[\left[\alpha_{1}, \alpha_{2}\right]\right]\left(\gamma_{1,2}, e_{3}\right)^{(1)} \oplus \mathcal{R}\left[\left[\alpha_{1}, \alpha_{2}\right]\right]\left(\gamma_{1,2}, e_{3}\right)^{(2)}
\end{aligned}
$$$$
\text { c) }
$$$$
\text { d) }
$$

Recombining terms gives

$$
\begin{aligned}
& \mathcal{R} \oplus \mathcal{R}\left[\left[\alpha_{2}\right]\right] \alpha_{2} \oplus \mathcal{R}\left[\left[\alpha_{1}, \alpha_{2}\right]\right] \alpha_{1} \\
& \oplus \mathcal{R}\left[\left[\alpha_{1}, \alpha_{2}\right]\right] \gamma_{1,2} \otimes \mathbb{R} e_{3} \\
& =\mathcal{R}\left[\left[\alpha_{1}, \alpha_{2}\right]\right] e_{3} \oplus \mathcal{R}\left[\left[\alpha_{2}\right]\right]\left(\alpha_{2}, e_{3}\right)^{(1)} \\
& \oplus \mathcal{R}\left[\left[\alpha_{2}\right]\right]\left(\alpha_{2}, e_{3}\right)^{(2)} \oplus \mathcal{R}\left[\left[\alpha_{1}, \alpha_{2}\right]\right]\left(\alpha_{1}, e_{3}\right)^{(1)} \\
& \oplus \mathcal{R}\left[\left[\alpha_{1}, \alpha_{2}\right]\right]\left(\alpha_{1}, e_{3}\right)^{(2)} \oplus \mathcal{R}\left[\left[\alpha_{1}, \alpha_{2}\right]\right] \gamma_{1,2} e_{3} \\
& \oplus \mathcal{R}\left[\left[\alpha_{1}, \alpha_{2}\right]\right]\left(\gamma_{1,2}, e_{3}\right)^{(1)} \oplus \mathcal{R}\left[\left[\alpha_{1}, \alpha_{2}\right]\right]\left(\gamma_{1,2}, e_{3}\right)^{(2)} .
\end{aligned}
$$

Case 2: Similarly we have, 


$$
\begin{aligned}
& \mathcal{R} \oplus \mathcal{R}\left[\left[\alpha_{2}\right]\right] \alpha_{2} \oplus \mathcal{R}\left[\left[\alpha_{1}, \alpha_{2}\right]\right] \alpha_{1} \\
& \oplus \mathcal{R}\left[\left[\alpha_{1}, \alpha_{2}\right]\right] \gamma_{1,2} \otimes \mathbb{R} e_{6} \\
& =\mathcal{R}\left[\left[\alpha_{1}, \alpha_{2}\right]\right] e_{6} \oplus \mathcal{R}\left[\left[\alpha_{2}\right]\right]\left(\alpha_{2}, e_{6}\right)^{(1)} \\
& \oplus \mathcal{R}\left[\left[\alpha_{2}\right]\right]\left(\alpha_{2}, e_{6}\right)^{(2)} \oplus \mathcal{R}\left[\left[\alpha_{1}, \alpha_{2}\right]\right]\left(\alpha_{1}, e_{6}\right)^{(1)} \\
& \oplus \mathcal{R}\left[\left[\alpha_{1}, \alpha_{2}\right]\right]\left(\alpha_{1}, e_{6}\right)^{(2)} \oplus \mathcal{R}\left[\left[\alpha_{1}, \alpha_{2}\right]\right] \gamma_{1,2} e_{6} \\
& \oplus \mathcal{R}\left[\left[\alpha_{1}, \alpha_{2}\right]\right]\left(\gamma_{1,2}, e_{6}\right)^{(1)} \oplus \mathcal{R}\left[\left[\alpha_{1}, \alpha_{2}\right]\right]\left(\gamma_{1,2}, e_{6}\right)^{(2)} .
\end{aligned}
$$

Adding terms in case 1 and 2 we obtain:

$$
\begin{aligned}
& \text { ker } \mathrm{X}_{33} \\
& =\mathcal{R}\left[\left[\alpha_{1}, \alpha_{2}\right]\right] e_{3} \oplus \mathcal{R}\left[\left[\alpha_{1}, \alpha_{2}\right]\right]\left(\alpha_{1}, e_{3}\right)^{(1)} \\
& \oplus \mathcal{R}\left[\left[\alpha_{1}, \alpha_{2}\right]\right]\left(\alpha_{1}, e_{3}\right)^{(2)} \oplus \mathcal{R}\left[\left[\alpha_{2}\right]\right]\left(\alpha_{2}, e_{3}\right)^{(1)} \\
& \oplus \mathcal{R}\left[\left[\alpha_{2}\right]\right]\left(\alpha_{2}, e_{3}\right)^{(2)} \oplus \mathcal{R}\left[\left[\alpha_{1}, \alpha_{2}\right]\right] \gamma_{1,2} e_{3} \\
& \oplus \mathcal{R}\left[\left[\alpha_{1}, \alpha_{2}\right]\right]\left(\gamma_{1,2}, e_{3}\right)^{(1)} \oplus \mathcal{R}\left[\left[\alpha_{1}, \alpha_{2}\right]\right]\left(\gamma_{1,2}, e_{3}\right)^{(2)} \\
& \oplus \mathcal{R}\left[\left[\alpha_{1}, \alpha_{2}\right]\right] e_{6} \oplus \mathcal{R}\left[\left[\alpha_{1}, \alpha_{2}\right]\right]\left(\alpha_{1}, e_{6}\right)^{(1)} \\
& \oplus \mathcal{R}\left[\left[\alpha_{1}, \alpha_{2}\right]\right]\left(\alpha_{1}, e_{6}\right)^{(2)} \oplus \mathcal{R}\left[\left[\alpha_{2}\right]\right]\left(\alpha_{2}, e_{6}\right)^{(1)} \\
& \oplus \mathcal{R}\left[\left[\alpha_{2}\right]\right]\left(\alpha_{2}, e_{6}\right)^{(2)} \oplus \mathcal{R}\left[\left[\alpha_{1}, \alpha_{2}\right]\right] \gamma_{1,2} e_{6} \\
& \oplus \mathcal{R}\left[\left[\alpha_{1}, \alpha_{2}\right]\right]\left(\gamma_{1,2}, e_{6}\right)^{(1)} \oplus \mathcal{R}\left[\left[\alpha_{1}, \alpha_{2}\right]\right]\left(\gamma_{1,2}, e_{6}\right)^{(2)} .
\end{aligned}
$$

Finally, to complete the calculation, it is necessary to compute the transvectants that appear. These are of the form $\left(f, e_{3}\right)^{(i)}$ and $\left(f, e_{6}\right)^{(i)}$ for $i=0,1,2$ where $f=\left\{\alpha_{1}, \alpha_{2}, \gamma_{1,2}\right\}$.

$$
\left(f, e_{3}\right)^{(0)}=\left[\begin{array}{l}
0 \\
0 \\
f
\end{array}\right]
$$

$$
\begin{aligned}
\left(f, e_{3}\right)^{(1)}=w_{f} f\left[\begin{array}{l}
0 \\
-1 \\
0
\end{array}\right]-\left[\begin{array}{l}
0 \\
0 \\
2 \mathcal{Y} f
\end{array}\right]=\left[\begin{array}{c}
0 \\
-1 w_{f} f \\
-2 \mathcal{Y} f
\end{array}\right]=\left[\begin{array}{c}
0 \\
-\mathcal{X Y} f \\
-2 \mathcal{Y} f
\end{array}\right] \\
\left(f, e_{3}\right)^{(2)}=w_{f}\left(w_{f}-1\right) \mathcal{Y} f\left(M^{*}\right)^{2} e_{3} \\
-2\left(w_{f}-1\right) \mathcal{Y} f M^{*} e_{3}+2 \mathcal{Y}^{2} f e_{3} \\
=-2\left[\begin{array}{c}
\mathcal{X}^{2} \mathcal{Y}^{2} f \\
\mathcal{X} \mathcal{Y}^{2} f \\
\mathcal{Y}^{2} f
\end{array}\right] .
\end{aligned}
$$

We ignore the nonzero constants -1 and -2 because we are concerned with computing basis elements. For the basis $e_{6}$ we have:

$$
\begin{aligned}
\left(f, e_{6}\right)^{(0)} & =\left[\begin{array}{l}
0 \\
0 \\
0 \\
0 \\
0 \\
f
\end{array}\right], \quad\left(f, e_{6}\right)^{(1)}=\left[\begin{array}{c}
0 \\
0 \\
0 \\
0 \\
\mathcal{X Y} f \\
\mathcal{Y} f
\end{array}\right] \\
\left(f, e_{6}\right)^{(2)} & =\left[\begin{array}{c}
0 \\
0 \\
0 \\
\mathcal{X}^{2} \mathcal{Y}^{2} f \\
\mathcal{X} \mathcal{Y}^{2} f \\
\mathcal{Y}^{2} f
\end{array}\right] .
\end{aligned}
$$

Therefore the normal form for system with linear part $N_{33}$ is:

$$
\begin{gathered}
\left.\operatorname{ker} \mathrm{X}_{33}=\mathcal{R}\left[\left[\alpha_{1}, \alpha_{2}, \beta_{1}, \beta_{2}, \xi_{1,2}\right]\right]\left[\begin{array}{l}
0 \\
0 \\
1 \\
0 \\
0 \\
0
\end{array}\right] \oplus \mathcal{R}\left[\left[\alpha_{1}, \alpha_{2}, \beta_{1}, \beta_{2}, \xi_{1,2}\right]\right]\right]\left[\begin{array}{c}
0 \\
\mathcal{X} \mathcal{Y} \alpha_{1} \\
\mathcal{Y} \alpha_{1} \\
0 \\
0 \\
0
\end{array}\right] \oplus \mathcal{R}\left[\left[\alpha_{1}, \alpha_{2}, \beta_{1}, \beta_{2}, \xi_{1,2}\right]\right]\left[\begin{array}{c}
\mathcal{X}^{2} \mathcal{Y}^{2} \alpha_{1} \\
\mathcal{X} \mathcal{Y}^{2} \alpha_{1} \\
\mathcal{Y}^{2} \alpha_{1} \\
0 \\
0 \\
0
\end{array}\right] \\
\oplus \mathcal{R}\left[\left[\alpha_{2}, \beta_{1}, \beta_{2}, \xi_{1,2}\right]\right]\left[\begin{array}{l}
0 \\
\mathcal{X} \mathcal{Y} \alpha_{2} \\
\mathcal{Y} \alpha_{2} \\
0 \\
0 \\
0
\end{array}\right] \oplus \mathcal{R}\left[\left[\alpha_{2}, \beta_{1}, \beta_{2}, \xi_{1,2}\right]\right]\left[\begin{array}{c}
\mathcal{X}^{2} \mathcal{Y}^{2} \alpha_{2} \\
\mathcal{X} \mathcal{Y}^{2} \alpha_{2} \\
\mathcal{Y}^{2} \alpha_{2} \\
0 \\
0 \\
0
\end{array}\right]
\end{gathered}
$$




$$
\begin{aligned}
& \mathcal{R}\left[\left[\alpha_{1}, \alpha_{2}, \beta_{1}, \beta_{2}, \xi_{1,2}\right]\right]\left[\begin{array}{l}
0 \\
0 \\
\gamma_{12} \\
0 \\
0 \\
0
\end{array}\right] \oplus \mathcal{R}\left[\left[\alpha_{1}, \alpha_{2}, \beta_{1}, \beta_{2}, \xi_{1,2}\right]\right]\left[\begin{array}{c}
0 \\
\mathcal{X} \gamma_{1,2} \\
\mathcal{Y} \gamma_{1,2} \\
0 \\
0 \\
0
\end{array}\right] \oplus \mathcal{R}\left[\left[\alpha_{1}, \alpha_{2}, \beta_{1}, \beta_{2}, \xi_{1,2}\right]\right]\left[\begin{array}{c}
\mathcal{X}^{2} \mathcal{Y}^{2} \gamma_{1,2} \\
\mathcal{X} \mathcal{Y}^{2} \gamma_{1,2} \\
\mathcal{Y}^{2} \gamma_{1,2} \\
0 \\
0 \\
0
\end{array}\right] \\
& \oplus \mathcal{R}\left[\left[\alpha_{1}, \alpha_{2}, \beta_{1}, \beta_{2}, \xi_{1,2}\right]\right]\left[\begin{array}{l}
0 \\
0 \\
0 \\
0 \\
0 \\
1
\end{array}\right] \oplus \mathcal{R}\left[\left[\alpha_{1}, \alpha_{2}, \beta_{1}, \beta_{2}, \xi_{1,2}\right]\right]\left[\begin{array}{c}
0 \\
0 \\
0 \\
0 \\
\mathcal{X} \mathcal{Y} \alpha_{1} \\
\mathcal{Y} \alpha_{1}
\end{array}\right] \oplus \mathcal{R}\left[\left[\alpha_{1}, \alpha_{2}, \beta_{1}, \beta_{2}, \xi_{1,2}\right]\right]\left[\begin{array}{c}
0 \\
0 \\
0 \\
\mathcal{X}^{2} \mathcal{Y}^{2} \alpha_{1} \\
\mathcal{X} \mathcal{Y}^{2} \alpha_{1} \\
\mathcal{Y}^{2} \alpha_{1}
\end{array}\right] \\
& \oplus \mathcal{R}\left[\left[\alpha_{2}, \beta_{1}, \beta_{2}, \xi_{1,2}\right]\right]\left[\begin{array}{c}
0 \\
0 \\
0 \\
0 \\
\mathcal{X} \mathcal{Y} \alpha_{2} \\
\mathcal{Y} \alpha_{2}
\end{array}\right] \oplus \mathcal{R}\left[\left[\alpha_{2}, \beta_{1}, \beta_{2}, \xi_{1,2}\right]\right]\left[\begin{array}{c}
0 \\
0 \\
0 \\
\mathcal{X}^{2} \mathcal{Y}^{2} \alpha_{2} \\
\mathcal{X} \mathcal{Y}^{2} \alpha_{2} \\
\mathcal{Y}^{2} \alpha_{2}
\end{array}\right] \oplus \mathcal{R}\left[\left[\alpha_{1}, \alpha_{2}, \beta_{1}, \beta_{2}, \xi_{1,2}\right]\right]\left[\begin{array}{l}
0 \\
0 \\
0 \\
0 \\
0 \\
\gamma_{1,2}
\end{array}\right] \\
& \oplus \mathcal{R}\left[\left[\alpha_{1}, \alpha_{2}, \beta_{1}, \beta_{2}, \xi_{1,2}\right]\right]\left[\begin{array}{c}
0 \\
0 \\
0 \\
0 \\
\mathcal{X} \mathcal{Y}_{1,2} \\
\mathcal{Y} \gamma_{1,2}
\end{array}\right] \oplus \mathcal{R}\left[\left[\alpha_{1}, \alpha_{2}, \beta_{1}, \beta_{2}, \xi_{1,2}\right]\right]\left[\begin{array}{c}
0 \\
0 \\
0 \\
\mathcal{X}^{2} \mathcal{Y}^{2} \gamma_{1,2} \\
\mathcal{X} \mathcal{Y}^{2} \gamma_{1,2} \\
\mathcal{Y}^{2} \gamma_{1,2}
\end{array}\right] .
\end{aligned}
$$

\subsection{System with Linear Part $N_{333}$}

The Stanley decomposition for ring of invariants of a system with linear part $N_{333}$ is given by:

$$
\begin{aligned}
& \operatorname{ker} \mathcal{X}_{333}= \mathbb{R}\left[\left[\alpha_{1}, \alpha_{2}, \alpha_{3}, \beta_{1}, \beta_{2}, \beta_{3}, \xi_{1,2}, \xi_{1,3}\right]\right] \\
& \oplus \mathbb{R}\left[\left[\alpha_{1}, \alpha_{2}, \alpha_{3}, \beta_{1}, \beta_{2}, \beta_{3}, \xi_{1,2}, \xi_{1,3}\right]\right] \gamma_{1,2} \\
& \oplus \mathbb{R}\left[\left[\alpha_{1}, \alpha_{2}, \alpha_{3}, \beta_{1}, \beta_{2}, \beta_{3}, \xi_{1,2}, \xi_{1,3}\right]\right] \gamma_{1,3} \\
& \oplus \mathbb{R}\left[\left[\alpha_{1}, \alpha_{2}, \alpha_{3}, \beta_{1}, \beta_{2}, \beta_{3}, \xi_{1,2}, \xi_{1,3}\right]\right] \gamma_{1,2} \gamma_{1,3} \\
& \oplus \mathbb{R}\left[\left[\alpha_{2}, \alpha_{3}, \beta_{1}, \beta_{2}, \beta_{3}, \xi_{1,2}, \xi_{1,3}, \xi_{2,3}\right]\right] \gamma_{2,3} \\
& \oplus \mathbb{R}\left[\left[\alpha_{2}, \alpha_{3}, \beta_{1}, \beta_{2}, \beta_{3}, \xi_{1,2}, \xi_{1,3}, \xi_{2,3}\right]\right] \xi_{2,3} \\
& \oplus \mathbb{R}\left[\left[\alpha_{2}, \alpha_{3}, \beta_{1}, \beta_{2}, \beta_{3}, \xi_{1,2}, \xi_{1,3}, \xi_{2,3}\right]\right] \gamma_{1,2} \gamma_{2,3} \\
& \oplus \mathbb{R}\left[\left[\alpha_{2}, \alpha_{3}, \beta_{1}, \beta_{2}, \beta_{3}, \xi_{1,2}, \xi_{1,3}, \xi_{2,3}\right]\right] \gamma_{1,2} \xi_{2,3} \\
& \oplus \mathbb{R}\left[\left[\alpha_{3}, \beta_{1}, \beta_{2}, \beta_{3}, \xi_{1,2}, \xi_{1,3}, \xi_{2,3}\right]\right]\left(\gamma_{1,2}, \alpha_{3}\right)^{(1)} \\
& \oplus \mathbb{R}\left[\left[\alpha_{3}, \beta_{1}, \beta_{2}, \beta_{3}, \xi_{1,2}, \xi_{1,3}, \xi_{2,3}\right]\right]\left(\gamma_{1,2}, \alpha_{3}\right)^{(2)}
\end{aligned}
$$

(see [6]).

The basis elements for ker $\mathcal{X}_{333}$ are $e_{3}, e_{6}$ and $e_{9}$. Therefore we need to compute the box product of the invariants ring ker $\mathcal{X}_{333}$ with $\mathbb{R} e_{3} \oplus \mathbb{R} e_{6} \oplus \mathbb{R} e_{9}$. Thus ker $\mathrm{X}_{333}=\operatorname{ker} \mathcal{X}_{333} \otimes\left[\mathbb{R} e_{3} \oplus \mathbb{R} e_{6} \oplus \mathbb{R} e_{9}\right]$. Let $\mathcal{R}=\left[\left[\beta_{1}, \beta_{2}, \beta_{3}, \xi_{1,2}, \xi_{1,3}\right]\right]$, then

ker $\mathrm{X}_{333}$

$=\left[\mathcal{R}\left[\left[\alpha_{1}, \alpha_{2}, \alpha_{3}\right]\right] \oplus \mathcal{R}\left[\left[\alpha_{1}, \alpha_{2}, \alpha_{3}\right]\right] \gamma_{1,2}\right.$ $\oplus \mathcal{R}\left[\left[\alpha_{1}, \alpha_{2}, \alpha_{3}\right]\right] \gamma_{1,3} \oplus \mathcal{R}\left[\left[\alpha_{1}, \alpha_{2}, \alpha_{3}\right]\right] \gamma_{1,2} \gamma_{1,3}$ $\oplus \mathcal{R}\left[\left[\alpha_{2}, \alpha_{3}, \xi_{2,3}\right]\right] \gamma_{2,3} \oplus \mathcal{R}\left[\left[\alpha_{2}, \alpha_{3}, \xi_{2,3}\right]\right] \xi_{2,3}$ $\oplus \mathcal{R}\left[\left[\alpha_{2}, \alpha_{3}, \xi_{2,3}\right]\right] \gamma_{1,2} \gamma_{2,3} \oplus \mathcal{R}\left[\left[\alpha_{2}, \alpha_{3}, \xi_{2,3}\right]\right] \gamma_{1,2} \xi_{2,3}$ $\left.\oplus \mathcal{R}\left[\left[\alpha_{3}, \xi_{2,3}\right]\right]\left(\alpha_{3}, \gamma_{1,2}\right)^{(1)} \oplus \mathcal{R}\left[\left[\alpha_{3}, \xi_{2,3}\right]\right]\left(\alpha_{3}, \gamma_{1,2}\right)^{(2)}\right]$ $\otimes\left[\mathbb{R} e_{3} \oplus \mathbb{R} e_{6} \oplus \mathbb{R} e_{9}\right]$

There are three cases to consider. Computing and simplifying the cases we obtain the normal form as: 


$$
\begin{aligned}
& \text { ker } \mathbf{X}_{333} \\
& =\mathcal{R}\left[\left[\alpha_{1}, \alpha_{2}, \alpha_{3}\right]\right] e_{3(n)} \oplus \mathcal{R}\left[\left[\alpha_{3}\right]\right]\left(\alpha_{3}, e_{3}(n)\right)^{(i)} \oplus \mathcal{R}\left[\left[\alpha_{2}, \alpha_{3}\right]\right]\left(\alpha_{2}, e_{3}(n)\right)^{(i)} \oplus \mathcal{R}\left[\left[\alpha_{1}, \alpha_{2}, \alpha_{3}\right]\right]\left(\alpha_{1}, e_{3}(n)\right)^{(i)} \\
& \oplus \mathcal{R}\left[\left[\alpha_{1}, \alpha_{2}, \alpha_{3}\right]\right] \gamma_{1,2} e_{3}(n) \oplus \mathcal{R}\left[\left[\alpha_{1}, \alpha_{2}, \alpha_{3}\right]\right]\left(\alpha_{1} \gamma_{1,2}, e_{3}(n)\right)^{(i)} \oplus \mathcal{R}\left[\left[\alpha_{2}, \alpha_{3}\right]\right]\left(\alpha_{2} \gamma_{1,2}, e_{3}(n)\right)^{(i)} \oplus \mathcal{R}\left[\left[\alpha_{3}\right]\right]\left(\gamma_{1,2}, e_{3}(n)\right)^{(i)} \\
& \oplus \mathcal{R}\left[\left[\alpha_{1}, \alpha_{2}, \alpha_{3}\right]\right] \gamma_{1,3} e_{3^{(n)}} \oplus \mathcal{R}\left[\left[\alpha_{1}, \alpha_{2}, \alpha_{3}\right]\right]\left(\alpha_{1} \gamma_{1,3}, e_{3}(n)\right)^{(i)} \oplus \mathcal{R}\left[\left[\alpha_{2}, \alpha_{3}\right]\right]\left(\alpha_{2} \gamma_{1,3}, e_{3}(n)\right)^{(i)} \oplus \mathcal{R}\left[\left[\alpha_{3}\right]\right]\left(\gamma_{1,3}, e_{3}(n)\right)^{(i)} \\
& \oplus \mathcal{R}\left[\left[\alpha_{1}, \alpha_{2}, \alpha_{3}\right]\right] \gamma_{1,2} \gamma_{1,3} e_{3}(n) \oplus \mathcal{R}\left[\left[\alpha_{1}, \alpha_{2}, \alpha_{3}\right]\right]\left(\alpha_{1} \gamma_{1,2} \gamma_{1,3}, e_{3}(n)\right)^{(i)} \oplus \mathcal{R}\left[\left[\alpha_{2}, \alpha_{3}\right]\right]\left(\alpha_{2} \gamma_{1,2} \gamma_{1,3}, e_{3}(n)\right)^{(i)} \\
& \oplus \mathcal{R}\left[\left[\alpha_{3}\right]\right]\left(\gamma_{1,2} \gamma_{1,3}, e_{3}(n)\right)^{(i)} \oplus \mathcal{R}\left[\left[\alpha_{2}, \alpha_{3}, \xi_{2,3}\right]\right] \gamma_{2,3} e_{3}(n) \oplus \mathcal{R}\left[\left[\alpha_{3}, \xi_{2,3}\right]\right]\left(\alpha_{3} \gamma_{2,3}, e_{3}(n)\right)^{(i)} \\
& \oplus \mathcal{R}\left[\left[\alpha_{2}, \alpha_{3}, \xi_{2,3}\right]\right]\left(\alpha_{2} \gamma_{2,3}, e_{3}(n)\right)^{(i)} \oplus \mathcal{R}\left[\left[\alpha_{2}, \alpha_{3}, \xi_{2,3}\right]\right] \xi_{2,3} e_{3}(n) \oplus \mathcal{R}\left[\left[\alpha_{3}, \xi_{2,3}\right]\right]\left(\alpha_{3} \xi_{2,3}, e_{3}(n)\right)^{(i)} \\
& \oplus \mathcal{R}\left[\left[\alpha_{2}, \alpha_{3}, \xi_{2,3}\right]\right]\left(\alpha_{2} \xi_{2,3}, e_{3}(n)\right)^{(i)} \oplus \mathcal{R}\left[\left[\alpha_{2}, \alpha_{3}, \xi_{2,3}\right]\right] \gamma_{1,2} \gamma_{2,3} e_{3}(n) \oplus \mathcal{R}\left[\left[\alpha_{3}, \xi_{2,3}\right]\right]\left(\alpha_{3} \gamma_{1,2} \gamma_{2,3}, e_{3}(n)\right)^{(i)}
\end{aligned}
$$

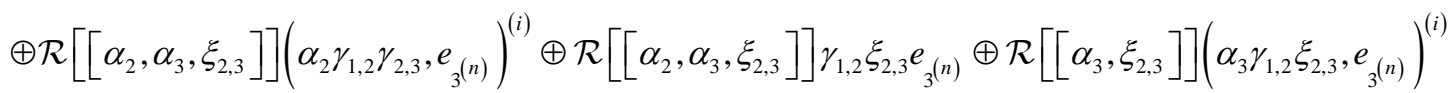

$$
\begin{aligned}
& \oplus \mathcal{R}\left[\left[\alpha_{2}, \alpha_{3}, \xi_{2,3}\right]\right]\left(\alpha_{2} \gamma_{1,2} \xi_{2,3}, e_{3^{(n)}}\right)^{(i)} \oplus \mathcal{R}\left(\gamma_{1,2}, \alpha_{3}\right)^{(1)} e_{3^{(n)}} \oplus \mathcal{R}\left[\left[\alpha_{3}, \xi_{2,3}\right]\right]\left(\left(\gamma_{1,2}, \alpha_{3}\right)^{(1)}, e_{3^{(n)}}\right)^{(i)} \\
& \oplus \mathcal{R}\left(\gamma_{1,2}, \alpha_{3}\right)^{(2)} e_{3(n)} \oplus \mathcal{R}\left[\left[\alpha_{3}, \xi_{2,3}\right]\right]\left(\left(\gamma_{1,2}, \alpha_{3}\right)^{(2)}, e_{3(n)}\right)^{(i)}
\end{aligned}
$$

where $i=1,2$ and $n=1,2,3$ such that $e_{3^{(1)}}=e_{3}$, $e_{3(2)}=e_{6}$ and $e_{3(3)}=e_{9}$

In general, from the above examples we conclude that the normal forms are obtained by computing the box product

$$
\operatorname{ker} \mathrm{X}=\operatorname{ker} \mathcal{X} \otimes \mathbb{R}_{3}{ }_{3}(n) \text {. }
$$

The basis of the normal form of ker $\mathrm{X}$ are transvectants of the form: $\left(f, e_{3}(n)\right)^{(i)}$ where $f$ is the standard monomials of Stanley decomposition of the ring of invariants, $\operatorname{ker} \mathcal{X}, i=0,1,2$ and $n=1, \cdots, n$.

As an example we find the normal form for a system with linear part $N_{3}$, we first find the ring of invariants ker $\mathcal{X}_{3}$ where $\mathcal{X}=2 x \frac{\partial}{\partial y}+2 y \frac{\partial}{\partial z}$ using $x, y, z$. By inspection $\alpha=x$ and $\beta=y^{2}-x z$, and this generates the entire ring; that is

$$
\text { ker } \mathcal{X}_{3}=\mathbb{R}[\alpha, \beta]
$$

To check this, we note that the weight of $\alpha$ is two and $\beta$ is of weight zero, so the table function of $\mathbb{R}[\alpha, \beta]$ is

$$
T=\frac{1}{\left(1-d w^{2}\right)\left(1-d^{2}\right)}
$$

Hence

$$
\left.\frac{\partial}{\partial w}\left(T_{3}\right)\right|_{w=1}=\frac{1}{(1-d)^{3}},
$$

this implies (0.1).

The next step is to compute $\operatorname{ker} \mathrm{X}$ as a module over ker $\mathcal{X}_{3} . N_{3}$ contains one Jordan block of size 3 hence the differential operators

$$
\begin{aligned}
& \mathcal{X}=2 x \frac{\partial}{\partial y}+2 y \frac{\partial}{\partial z} \\
& \mathcal{Y}=y \frac{\partial}{\partial x}+z \frac{\partial}{\partial y} .
\end{aligned}
$$

In this case the basis elements is $e_{3}$ which is of weight 2 therefore the normal form is

$$
\begin{aligned}
\operatorname{ker} \mathrm{X}_{3} & =\mathbb{R}[[\alpha, \beta]] e_{3} \oplus \mathbb{R}[[\alpha, \beta]]\left(\alpha, e_{3}\right)^{(1)} \\
& \oplus \mathbb{R}[[\alpha, \beta]]\left(\alpha, e_{3}\right)^{(2)} \\
\operatorname{ker~}_{3} & =\mathbb{R}[[\alpha, \beta]]\left[\begin{array}{l}
0 \\
0 \\
1
\end{array}\right] \oplus \mathbb{R}[[\alpha, \beta]]\left[\begin{array}{c}
0 \\
\mathcal{X} \mathcal{Y} \alpha \\
\mathcal{Y} \alpha
\end{array}\right] \\
& \oplus \mathbb{R}[[\alpha, \beta]]\left[\begin{array}{l}
\mathcal{X}^{2} \mathcal{Y}^{2} \alpha \\
\mathcal{X} \mathcal{Y}^{2} \alpha \\
\mathcal{Y}^{2} \alpha
\end{array}\right]
\end{aligned}
$$




$$
\begin{aligned}
& {\left[\begin{array}{c}
\dot{x} \\
\dot{y} \\
\dot{z}
\end{array}\right]=\left[\begin{array}{ccc}
0 & 1 & 0 \\
0 & 0 & 1 \\
0 & 0 & 0
\end{array}\right]\left[\begin{array}{l}
x \\
y \\
z
\end{array}\right]+f_{1}(\alpha, \beta)\left[\begin{array}{l}
0 \\
0 \\
1
\end{array}\right]} \\
& +f_{2}(\alpha, \beta)\left[\begin{array}{c}
0 \\
\mathcal{X} \mathcal{Y} \alpha \\
\mathcal{Y} \alpha
\end{array}\right]+f_{3}(\alpha, \beta)\left[\begin{array}{l}
\mathcal{X}^{2} \mathcal{Y}^{2} \alpha \\
\mathcal{X Y}^{2} \alpha \\
\mathcal{Y}^{2} \alpha
\end{array}\right]
\end{aligned}
$$

We compute:

$$
\begin{gathered}
\mathcal{Y} \alpha=\mathcal{Y} x=y, \frac{1}{2} \mathcal{X Y} \alpha=x \\
\mathcal{Y}^{2} \alpha=z, \frac{1}{2} \mathcal{X} \mathcal{Y}^{2} \alpha=y, \frac{1}{4} \mathcal{X}^{2} \mathcal{Y}^{2} \alpha=x \\
{\left[\begin{array}{c}
\dot{x} \\
\dot{y} \\
\dot{z}
\end{array}\right]=\left[\begin{array}{lll}
0 & 1 & 0 \\
0 & 0 & 1 \\
0 & 0 & 0
\end{array}\right]\left[\begin{array}{l}
x \\
y \\
z
\end{array}\right]+f_{1}(\alpha, \beta)\left[\begin{array}{l}
0 \\
0 \\
1
\end{array}\right]} \\
+f_{2}(\alpha, \beta)\left[\begin{array}{l}
0 \\
x \\
y
\end{array}\right]+f_{3}(\alpha, \beta)\left[\begin{array}{l}
x \\
y \\
z
\end{array}\right]
\end{gathered}
$$

The differential equations in $\operatorname{sl}(2)$ normal form are:

$$
\begin{aligned}
\dot{x} & =y+h\left(x, y^{2}-x z\right) x=y+\left(\gamma_{1} x+\gamma_{2} x^{2}\right) x \\
= & y+\gamma_{1} x^{2}+\cdots \\
\dot{y} & =z+g\left(x, y^{2}-x z\right) x+h\left(x, y^{2}-x z\right) y \\
& =z+g(x) x+h(x) y \\
& =y+\left(\beta_{1} x+\beta_{2} x^{2}+\cdots\right) x+\left(\gamma_{1} x+\gamma_{2} x^{2}+\cdots\right) y \\
& =z+\beta_{1} x^{2}+\gamma_{1} x y+\cdots \\
\dot{z} & =f\left(x, y^{2}-x z\right) x+g\left(x, y^{2}-x z\right) y+h\left(x, y^{2}-x z\right) z \\
& =f(x) x+g(x) y+h(x) z \\
& =\left(\alpha_{1} x+\alpha_{2} x^{2}\right) x+\cdots+\left(\beta_{1} x+\beta_{2} x^{2}+\cdots\right) y \\
& +\left(\gamma_{1} x+\gamma_{2} x^{2}+\cdots\right) z \\
& =\alpha_{1} x^{2}+\beta_{1} x y+\gamma_{1} x z+\cdots
\end{aligned}
$$

The normal form upto quadratic term is:

$$
\left[\begin{array}{c}
\dot{x} \\
\dot{y} \\
\dot{z}
\end{array}\right]=\left[\begin{array}{lll}
0 & 1 & 0 \\
0 & 0 & 1 \\
0 & 0 & 0
\end{array}\right]\left[\begin{array}{l}
x \\
y \\
z
\end{array}\right]+\left[\begin{array}{c}
\alpha x^{2} \\
\beta x^{2}+\alpha x y \\
\gamma x^{2}+\beta x y+\alpha x z
\end{array}\right] .
$$

Remark: The normal form of a dynamical systems is a powerful tool in the study of stability and bifurcations analysis. From the practical point of view, only the normal form with perturbation (bifurcation) parameters is useful in analyzing physical or engineering problems. In this paper the computation of the normal form has been mainly restricted to systems which do not contain perturbation parameters by setting the parameters to zero to obtain the simplified normal form. Having found the normal form of the reduced system we shall then add unfolding terms to get a parametric normal form for bifurcation analysis.

\section{REFERENCES}

[1] R. Cushman, J. A. Sanders and N. White, "Normal Form for the (2;n)-Nilpotent Vector Field Using Invariant Theory," Physica D: Nonlinear Phenomena, Vol. 30, No. 3, 1988, pp. 399-412. doi:10.1016/0167-2789(88)90028-0

[2] D. M. Malonza "Normal Forms for Coupled Takens-Bogdanov Systems," Journal of Nonlinear Mathematical Physics, Vol. 11, No. 3, 2004, pp. 376-398. doi:10.2991/jnmp.2004.11.3.8

[3] W. W. Adams and P. Loustaunau, “An Introduction to Gröbner Bases,” American Mathematical Society, Providence, 1994.

[4] J. Murdock and J. A. Sanders, “A New Transvectant Algorithm for Nilpotent Normal Forms,” Journal of Differential Equations, Vol. 238, No. 1, 2007, pp. 234-256. doi:10.1016/j.jde.2007.03.016

[5] N. Sri NAmachchivaya, M. M. Doyle, W. F. Langford and N. W. Evans, "Normal Form for Generalized Hopf Bifurcation with Non-Semisimple 1:1 Resonance," Zeitschrift für Angewandte Mathematik und Physik (ZAMP), Vol. 45, No. 2, 1994, pp. 312-335. doi:10.1007/BF00943508

[6] G. Gachigua and D. Malonza, "Stanley Decomposition of Coupled $N_{333}$ System,” Proceedings of the 1st Kenyatta University International Mathematics Conference, Nairobi, 6-10 June 2011, pp. 39-52. 